VII International Congress of

Science, Technology,

Entrepreneurship and

Innovation (SECTEI 2020)

Corresponding Author:

P. Abdo-Peralta

paulitag_abdo@hotmail.com

Published: 26 August 2021

Production and Hosting by

Knowledge $E$

(c) P. Abdo-Peralta et al. This article is distributed under the terms of the Creative Commons Attribution License, which permits unrestricted use and redistribution provided that the original author and source are credited.

\section{Evaluation of Three Organic Products With Three Doses for Boat Control (Phytophthora infestans) in Potato Cultivation (Solanum tuberosum cv. Fripapa) Riobamba Canton, Chimborazo Province}

\section{Evaluación de Tres Productos Ecológicos con Tres Dosis Para el Control de Lancha (Phy- tophthora infestans) en el Cultivo de Papa (Solanum Tuberosum cv. Fripapa) Cantón Riobamba, Provincia de Chimborazo}

\author{
P. Abdo-Peralta, J. Haro, and K. Carrera
}

Escuela Superior Politécnica de Chimborazo

\section{Abstract}

This research proposes to evaluate three ecological products with three doses for the control disease (Phytophthora infestants) in the potato crop (Solanum tuberosum cv. Fripapa) in the Riobamba canton, Chimborazo province. Three ecological products helped: Citrubact, Fosficarben, and Bioplus in high medium and low doses recommended by the producers, making applications every two weeks in this crop, to determine the best product in both the $P$. infestans control and performance of growing marginal rate. A design of randomized complete blocks (RCBD) in bifactorial combinations with 9 treatments, 4 replications and a control were used. As a result, the lower incidence of the disease was obtained by Fosficarben treatment in high doses $(4 \mathrm{cc} / \mathrm{Lt}$ ) with $8.33 \%$, due to the component ion phosphite, which has a direct effect on the fungal metabolism. The same behaviour was observed in the intensity where Fosficarben in high doses (4cc/Lt) obtained $12,83 \%$, being the lower intensity of the research. The treatment with better performance was also Fosficarben in high doses with $21496,80 \mathrm{l}<\mathrm{h} / \mathrm{ha}$. According to the economic analysis the treatment composed by Citrubact in low-doses (Icc/Lt) got a higher marginal rate of return in relation to other treatments of $798.97 \%$ and a net income of 2631.28 USD and a variable cost, followed by the Bioplus treatmentdue in medium doses (4cc/Lt) with $765.14 \%$ of marginal rate. In conclusion, it is possible to control $P$. infestans in the potato crop by using ecological products, obtaining a good performance, a healthy product and economic income.

Keywords: Ecological products, potato crop, control disease.

\section{Resumen}

La presente investigación propone: evaluar tres productos ecológicos con tres dosis para el control de lancha (Phytophthora infestans) en el cultivo de papa (Solanum tuberosum cv. Fripapa) Cantón Riobamba, Provincia de Chimborazo. Ayudándonos de tres productos ecológicos: Citrubact. Fosficarben y Bioplus, en dosis alta media y baja recomendadas por las empresas productoras, realizando aplicaciones cada dos semanas en este cultivo, para determinar el mejor producto, tanto en el control de $P$. infestans, como en rendimiento 
del cultivo y tasa de retorno marginal. Se utilizó un diseño de bloques completos al azar (DBCA) en arreglo bifactorial combinatorio, con 9 tratamientos, 4 repeticiones y un testigo. Resultando que la menor incidencia de la enfermedad la obtuvo el tratamiento formado por Fosficarben en dosis alta $(4 \mathrm{cc} / \mathrm{It})$ con $8,33 \%$, esto se debe a que contiene ion fosfito, que ejerce un efecto directo sobre el metabolismo fúngico. El mismo comportamiento se pudo observar en la intensidad donde Fosficarben en dosis alta (4cc/lt) obtuvo $12,83 \%$ siendo la menor intensidad del estudio. El tratamiento con mayor rendimiento también fue Fosficarben en dosis alta (4cc/lt) con $21496,80 \mathrm{~kg} / \mathrm{ha}$. En al análisis económico el tratamiento formado por Citrubact en dosis baja (1 cc/lt) obtuvo una mayor tasa de retorno marginal en relación al resto de tratamientos de 798,97\%, con un beneficio neto de 2631,28 USD y un costo variable de 177,55 USD; debido a su dosis baja, es el más rentable de los tratamientos en estudio, seguido por el tratamiento conformado por Bioplus en dosis media (4 cc/lt) con 765,14\% de tasa de retorno marginal. En conclusión se puede controlar a $P$. infestans en el cultivo de papa utilizando productos ecológicos, obteniendo un buen rendimiento, un producto sano y generando ingresos económicos.

Palabras Clave: Productos ecológicos, cosecha de papas, control de enfermedades.

\section{Introducción}

A inicios del decenio de 1990 , casi la totalidad de las papas se producían y consumían en Europa, América del Norte y en los países de la antigua Unión Soviética. Desde entonces se ha producido un espectacular aumento de la producción y la demanda de papa en Asia, África y América Latina, donde la producción aumentó de menos de 30 millones de toneladas a principios del decenio de 1960 a más de 165 millones en 2010. En 2008, por primera vez, la producción de la papa del mundo en desarrollo excedía el del mundo desarrollado. China se ha convertido en el primer productor mundial de papa, y poco menos de una tercera parte de todas las papas hoy se cosechan en China y la India [1]. El cultivo de papa constituye una actividad económica importante también en Ecuador, las provincias con mayor producción de papa son: Carchi, Pichincha, Cotopaxi, Tungurahua y Chimborazo, quienes aportaron con el 83\% de la producción de todo el país. Las mayores extensiones de cultivo corresponden en su orden a Chimborazo (19,39\%), Carchi (18,96\%), Tungurahua (14,98\%), Cotopaxi (14,54\%) y Pichincha (10,09\%). El mayor rendimiento corresponde a Carchi con 14 13,62 tn-1. Las siembras y cosechas durante todo el año permiten abastecer suficientemente el consumo nacional [2].

En las provincias de la Sierra, ocupan el $65 \%$ de la tierra en el cultivo de papa. Carchi en el norte es la provincia más importante en su producción. El 61\% de las tierras cultivadas con cultivos transitorios son sembradas con papa. La producción promedio alcanza 160000 toneladas y el promedio por hectárea es de 12,5 toneladas, siendo mayor que el promedio nacional que es de 7,5 toneladas por hectárea [2]. La papa es 
uno de los cultivos más importantes de la región interandina, constituyendo una de las fuentes vegetales más nutritivas, debido a que su contenido en carbohidratos y proteínas es mucho más alto que el existente en los cereales, raíces y otros tubérculos, motivo por el cual en Ecuador hace parte de los productos que constituyen la canasta básica popular.

La superficie cosechada de Papa presenta una tasa media de crecimiento de -1,62\% entre 2002 y 2011 . En el 2011 se observa un decrecimiento de 1,44\%. Sin embargo, la producción presenta una tasa promedio de crecimiento de 5,23\% entre 2002 y 2011 , el 2011 registra una tasa de variación de $-12,35 \%$ respecto al año anterior. Los cultivos de papa se encuentran principalmente en la Región Sierra. En el 2011 las provincias de Chimborazo, Cotopaxi y Carchi sumaron el 65,3\% de la Superficie Total Cosechada de este producto. Carchi es la provincia de mayor cultivo de papa, con una participación del $31,61 \%$ a nivel nacional,seguido de Cotopaxi con $19,16 \%$ y Chimborazo con $14,12 \%$ de la producción nacional [2].

En el período 2002-2010, la producción de papa presentó una tasa de variación anual promedio de 7,43\%. El mayor volumen se cultivó en 2004 , cuando con un crecimiento de 8,28\% alcanzó 413.368 TM, a un rendimiento de 7,16 TM/Ha. En el 2010 casi la totalidad de la producción se produjo en la Sierra, que tuvo una participación de 99,88\%, mientras que el Oriente y la Costa registraron 0,09\% y 0,03\% de participación, respectivamente [2].

El tizón tardío o lancha de la papa (Phytophtora infestans) es la enfermedad más importante que afecta al cultivo y es la que produce mayores pérdidas económicas en todo el mundo. La infección se produce al descender las temperaturas e incrementarse la humedad, aunque también es necesario un aumento de las temperaturas para la germinación de las esporas del hongo. Los síntomas son unas manchas de color verde situadas cerca de los bordes de los foliolos, que evolucionan a color negro y se diseminan por los peciolos hacia el tallo [3].

En Ecuador también es el mayor problema sanitario del cultivo de papa y por ende de los agricultores es el tizón tardío Las condiciones climáticas imperantes en la sierra ecuatoriana son las más favorables para la lancha. La enfermedad puede atacar al follaje de las plantas desde la emergencia hasta la cosecha (Kromann, 2007)

La enfermedad puede destruir el follaje y los tallos de la papa en cualquier momento durante la estación del crecimiento de las plantas. Puede atacar además a los tubérculos de la papa, los cuales se pudren en los terrenos de cultivo o cuando se les almacena, transporta o vende en el mercado [3].

El tizón tardío puede destruir totalmente todas las plantas de una zona de cultivo al cabo de una o dos semanas cuando las condiciones climáticas son favorables y cuando no se aplica ningún método de control. No obstante, las pérdidas varían de un área a la otra y de año en año dependiendo de la temperatura y la humedad predominantes 
en ciertos períodos de la estación de crecimiento de las plantas y de los métodos de control empleados [3].

Phytophtora infestans sigue siendo una plaga difícil de controlar hoy en día. Hay muchas opciones en la agricultura para el control de daños en el follaje y las infecciones del tubérculo. Las papas crecen durante toda la temporada y se estima que los tubérculos dejan de crecer cuando el $75 \%$ del follaje de la planta es destruido. Esto también debe tenerse en cuenta en los cultivos, ya que significa que las plantas no tienen que ser $100 \%$ resistentes a la plaga (Zwankhuizen et al. 1998).

La plaga puede ser controlada mediante la limitación de la fuente de infección. Deberían plantarse semillas de buena calidad y descartarse las papas de la temporada anterior pues pueden actuar como fuentes de infección (Zwankhuizen, Govers, Zadoks, 1998).

El uso de fungicidas para el control de la plaga normalmente se utiliza de manera preventiva, tal vez en relación con las previsiones de la enfermedad. En variedades de papas susceptibles, a veces pueden ser necesarias aplicaciones semanales de fungicida. La aplicación temprana es más eficaz (Zwankhuizen, Govers, Zadoks, 1998).

En un estudio realizado por INIAP [2] en el Ecuador los agricultores utilizan el control químico como principal alternativa para el control de la lancha. En la región de Tungurahua, el promedio de aplicaciones por ciclo de cultivo fue de 3 . El número de aplicaciones promedio el Carchi es de 8.

En Chimborazo el número promedio de controles fue de 3. Los primeros fungicidas efectivos desarrollados para el control de tizón tardío de la papa fueron los cúpricos posteriormente aparecieron los bisditiocarbamatos éstos últimos son los más ampliamente utilizados, pero tienen graves problemas con respecto a la exposición del agricultor y al medio ambiente y finalmente otros compuestos llamados actualmente de contacto. Una nueva era comenzó con la aparición de los fungicidas sistémicos [4]

La limpieza del terreno puede reducir la contaminación, impidiendo que el patógeno se propague a los tubérculos. Esto normalmente involucra el suelo o mantillo amontonado alrededor de los tallos de la papa. También puede destruirse el follaje alrededor de 2 semanas antes de la cosecha. Esto puede hacerse a través de un herbicida de contacto o mediante el uso de ácido sulfúrico para quemar el follaje (Zwankhuizen, Govers, Zadoks, 1998).

El cultivo orgánico de la papa es una manera sana de obtener productos con una reducción de un 25 por ciento en los costos de inversión y al mismo tiempo un 30 por ciento en el incremento de la producción [5]. Se usa una mejor nutrición para reducir la incidencia de enfermedades, con los nutrientes en proporciones adecuadas se logra reactivar las defensas de la planta, además se acondiciona el suelo y otros procesos que consiguen que la papa se desarrolle sana y sin ningún tipo de fungicida que resulta un peligro para los humanos [5]. 
Existe una gran preocupación por el resurgimiento de nuevas poblaciones de $P$. infestans, por el desconocimiento de los agricultores sobre el uso inadecuado de fungicidas, el desconocimiento del mecanismo de acción de los ingredientes activos, la alta dosificación ha creado la rápida pérdida de sensibilidad o resistencia de los fungicidas y por tanto ineficacia en el control de $P$. infestans. Esto es especialmente cierto para los países en desarrollo, por lo tanto se deberían realizar acciones que mejoren las estrategias de manejo integrado de tizón tardío y faciliten los esfuerzos de los mejoradores, para en un futuro contar con nuevas variedades, que Tengan altos niveles de resistencia estables que puedan contrarrestar el incremento de los niveles de patogenicidad exhibidos por P. infestans (Turkensteen y Flier 2002).

Los Productos Ecológicos son productos que se obtienen de sistemas productivos sustentables [6].

Un producto ecológico se logra mediante el uso racional de los recursos naturales, la no utilización de productos de síntesis química, el incremento y/o mantenimiento de la fertilidad, el incremento y/o mantenimiento de la biodiversidad y el respaldo por medio de registros que avalen el manejo aplicado [6]

Un producto orgánico debe poseer identidad e integridad. Se sostiene que los sistemas de producción de la agricultura orgánica se deben obtener naturalmente de las rotaciones de los cultivos, de los residuos de cultivo del abono mineral, del abono verde, de las leguminosas, de minerales de roca, del laboreo mecanizado, del control biológico de pestes y reciclajes de deshechos, para mantener el suelo productivo y cultivable, aportando los nutrientes para el buen crecimiento de las plantas [6].

La agricultura orgánica es un estilo o un sistema de producción que formulado con base agroecológica evita usar productos que afecten al equilibrio del ecosistema silvoagropecuario, empleando para ello técnicas que incorporen las características específicas del medio rural y que, a su vez, estén de acuerdo con la realidad socioeconómica y cultural, con el objeto de hacer uso racional de los recursos renovables que derive en la obtención de una producción sostenida en el largo plazo y con un alto valor biológico para la salud humana [6].

Un producto orgánico debe poseer identidad e integridad. Se sostiene que los sistemas de producción de la agricultura orgánica se deben obtener naturalmente de las rotaciones de los cultivos, de los residuos de cultivo del abono mineral, del abono verde, de las leguminosas, de minerales de roca, del laboreo mecanizado, del control biológico de pestes y reciclajes de deshechos, para mantener el suelo productivo y cultivable, aportando los nutrientes para el buen crecimiento de las plantas [6].

La agricultura orgánica es un estilo o un sistema de producción que formulado con base agroecológica evita usar productos que afecten al equilibrio del ecosistema silvoagropecuario, empleando para ello técnicas que incorporen las características 
específicas del medio rural y que, a su vez, estén de acuerdo con la realidad socioeconómica y cultural, con el objeto de hacer uso racional de los recursos renovables que derive en la obtención de una producción sostenida en el largo plazo y con un alto valor biológico para la salud humana [6]. Pretende dar solución a los problemas del agro a partir de los propios recursos del agricultor librándolo de los productos químicos tóxicos [7].

La Agricultura Alternativa tomó fuerza a partir de la década de los 70, con el propósito de mejorar la relación entre el ser humano y la tierra, donde el punto de vista productivo, conservación de la biodiversidad, salud, el disfrute de los paisajes y naturaleza son aspectos fundamentales [7].

Al iniciar éste cambio, debemos entender que el suelo es la base para los cultivos y la vida, por lo que el mejoramiento de la fertilidad de los suelos con abonos orgánicos y el control adecuado de plagas y enfermedades es muy importante [7].

En la actualidad, muchos agricultores y técnicos practican y recomiendan el Manejo Integrado de Plagas, que es un sistema que incluye varios métodos y procesos, que combinados reducen al mínimo los daños causados por las plagas, enfermedades y malezas, evitando de esta manera el deterioro del ambiente. Estas técnicas son el resultado de la recopilación de las técnicas del conocimiento ancestral, que mediante el empleo de extractos vegetales propios de cada sector, pueden disminuir el uso de agroquímicos, cada vez más caros, más concentrados, y peligrosos, cuyo uso continuo ha provocado una mayor resistencia en los insectos y enfermedades, y ha eliminado a los enemigos naturales de las plagas [7].

Para realizar el presente estudio se requiere determinar el mejor producto ecológico para el control de lancha ( $P$. infestans) en el cultivo de papa (Solanum tuberosum cv. Fripapa); determinar la mejor dosis para su control y analizar el impacto económico en el uso comparativo de cada uno de los tratamientos.

\section{Metodología}

\subsection{Ubicación Geográfica}

Facultad de Recursos Naturales, ESPOCH, Altitud: 2778 m.s.n.m; Latitud: 141'05"'S.; Longitud: $78^{\circ} 40^{\prime} 20^{\prime \prime} \mathrm{W}$. Temperatura media anual: $13.5^{\circ} \mathrm{C}$; Humedad relativa: $56 \%$; Precipitación media anual: 405 mm; Clasificación Ecológica Según Holdrige (1982), la zona de formación ecológica estepa espinosa - Montano Bajo (ee-MB)

\subsection{Factores en Estudio}




\subsubsection{Variables independientes}

Los tratamientos ensayados fueron 10 incluido un testigo sin ninguna dosis de tratamiento, estos 229 se describen a continuación:

\section{Table 1}

Detalle de los tratamientos.

Tratamientos
T1
T2
T3
T4
T5
T6
T7
T8
T9
TA

\begin{tabular}{ll} 
Descripción & Dosis \\
\hline Fosficarben dosis alta & $4 \mathrm{cc} /$ Itro \\
\hline Fosficarben dosis media & $3 \mathrm{cc} /$ Itro \\
\hline Fosficarben dosis baja & $2 \mathrm{cc} /$ Itro \\
\hline Citrubact dosis alta & $3 \mathrm{cc} /$ tro \\
\hline Citrubact dosis media & $2 \mathrm{cc} /$ Itro \\
\hline Citrubact dosis baja & $1 \mathrm{cc} /$ Itro \\
\hline Bioplus dosis alta & $5 \mathrm{cc} /$ Itro \\
\hline Bioplus dosis media & $4 \mathrm{cc} /$ Itro \\
Bioplus dosis baja & $3 \mathrm{cc} /$ Itro \\
\hline Testigo absoluto & 0
\end{tabular}

\subsubsection{Variables dependientes}

- Incidencia de la enfermedad o Intensidad o Rendimiento en Tm/ha

- Relación Costo /Beneficio

\subsection{Unidad y Diseño Experimental}

Se utilizó un diseño de bloques completos al azar (DBCA) en arreglo bifactorial combinatorio, con 9 tratamientos, 4 repeticiones y un testigo. Se compararon tres productos, cada uno con tres dosis diferentes. De la combinación de los tratamientos y las repeticiones, dan 40 unidades experimentales. La parcela tenía forma rectangular con un largo de 50 metros y un ancho de $20 \mathrm{~m}$. El para total fue de $1000 \mathrm{~m} 2$.

La distancia de siembra entres surcos es de 0,8 $\mathrm{m}$ y entre plantas de 0,4 m. La distancia entre subparcelas fue de $1 \mathrm{~m}$. El área neta de cada parcela fue de 14,8 m2. La distancia entre bloques y parcelas fue de $1 \mathrm{~m}$. Para evitar el efecto de borde se eliminó 1 hilera y 4 plantas laterales por hilera.

En cada hilera se colocaron 12 plantas que por parcela daban un total de 48 plantas, todo el ensayo contó con 1920 plantas que se estudiaron al azar en un total de 400 . 


\subsection{Esquema del Análisis de Varianza}

Table 2

Análisis varianza elaboración: Abdo, 2012.

\begin{tabular}{l|l} 
Fuente de variacion (FV) & Grados de libertad (gl) \\
\hline Total & $\mathbf{3 9}$ \\
\hline Bloques & $\mathbf{3}$ \\
Tratamientos & $\mathbf{9}$ \\
Entre grupos & $\mathbf{3}$ \\
CO1 (combinación) & $\mathbf{1}$ \\
CO2 & $\mathbf{1}$ \\
CO3 & $\mathbf{1}$ \\
\hline DG1 (fosficarben) & $\mathbf{2}$ \\
PO1 (proyección ortogonal) & $\mathbf{1}$ \\
\hline PO2 & $\mathbf{1}$ \\
\hline DG2 (citrubact) & $\mathbf{2}$ \\
\hline PO1 & $\mathbf{1}$ \\
\hline PO2 & $\mathbf{1}$ \\
\hline DG3 (bioplus) & $\mathbf{2}$ \\
\hline PO1 & $\mathbf{1}$ \\
\hline PO2 & $\mathbf{1}$ \\
\hline ERROR & $\mathbf{2 7}$ \\
\hline
\end{tabular}

\subsection{Análisis Funcional}

- Prueba de Tukey 5\% para tratamientos

- Se determinó el coeficiente de variación CV

- Análisis económico mediante el método del CIMMYT

\section{Resultados y Discusión}

\subsection{Incidencia de la Enfermedad}

La incidencia de $P$. infestans, a los 88 días después de la siembra fue en promedio de $11,25 \%$ y un coeficiente de variación de $30,27 \%$ (Tabla 3), al someter los resultados experimentales al análisis de varianza no presentó diferencia significativa entre los productos, dosis, interacción y los diferentes contrastes.

Quince días después (103 días después de la siembra) se determinó una incidencia de $23,50 \%$ y un coeficiente de variación de 27,70\% (Tabla 3), al analizar los resultados 
experimentales según el ADEVA, presentó diferencia estadística al contrastar el Fosficarben frente a Citrubact y Bioplus de la misma manera cuando contrastamos la dosis media con la dosis baja (B2 vs B3) de Fosficarben.

La tercera (118 días después de la siembra) y cuarta lectura (133 días después de la siembra) de incidencia de $P$. infestans en el cultivo de papa en promedio registro $23,50 \%$ y un coeficiente de variación de $27,77 \%$ (Tabla 3), al someter los resultados experimentales al análisis de varianza se puede mencionar que se registró diferencia estadística $(p<0,05)$, para los contrastes entre los tratamientos $A 1$ vs $A 2 A 3$ y en B2 vs B3.

\section{Table 3}

Cuadrados medios para la incidencia de P. infestans del cultivo de papa (S. tuberosum CV. Fripapa).

\begin{tabular}{|c|c|c|c|c|c|c|c|c|c|}
\hline \multirow[b]{2}{*}{ F. Var } & \multirow[b]{2}{*}{$\mathrm{Gl}$} & \multicolumn{8}{|c|}{ Cuadrados medios de la incidencia cada 15 dias } \\
\hline & & $\begin{array}{c}\text { Incid } 1 \\
\text { (88 dias) }\end{array}$ & & $\begin{array}{c}\text { Incid } 2 \\
\text { (103 dias) }\end{array}$ & & $\begin{array}{c}\text { Incid } 3 \\
\text { (118 dias }\end{array}$ & & $\begin{array}{c}\text { Incid } 4 \\
\text { (133 dias }\end{array}$ & \\
\hline Total & 39 & & & & & & & & \\
\hline Bloques & 3 & 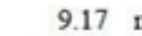 & ns & 216.67 & ** & 216.67 & ** & 216.67 & ** \\
\hline $\begin{array}{l}\text { Productos } \\
\text { A1 vs }\end{array}$ & 2 & 102.78 & ns & 136.11 & ns & 136.11 & ns & 136.11 & ns \\
\hline $\mathrm{A} 2 \mathrm{~A} 3$ & 1 & 138.89 & ns & 234.72 & * & 234.72 & * & 234.72 & * \\
\hline$A 2$ vs $A 3$ & 1 & 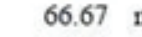 & ns & 37.50 & ns & 37.50 & ns & 37.50 & ns \\
\hline $\begin{array}{l}\text { Dosis } \\
\text { B1 vs }\end{array}$ & 2 & $52.78 \quad$ & ns & 102.78 & ns & 102.78 & ns & 102.78 & ns \\
\hline B2B3 & 1 & 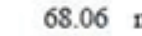 & ns & 138.89 & ns & 138.89 & ns & 138.89 & ns \\
\hline B2 vs B3 & 1 & $37.50 \mathrm{I}$ & ns & 66.67 & ns & 66.67 & ns & 66.67 & ns \\
\hline $\begin{array}{l}\text { Int } A B \\
\text { Fosficarben }\end{array}$ & 4 & $11.11 \mathrm{~g}$ & ns & 106.94 & ns & 106.94 & ns & 106.94 & ns \\
\hline$B 1$ vs $B 23$ & 1 & $4.17 \mathrm{I}$ & ns & 0.00 & ns & 0.00 & ns & 0.00 & ns \\
\hline $\begin{array}{l}\text { B2 vs B3 } \\
\text { Citrubact }\end{array}$ & 1 & $12.50 \mathrm{r}$ & ns & 200.00 & * & 200.00 & * & 200.00 & * \\
\hline$B 1$ vs $B 23$ & 1 & $66.67 \mathrm{I}$ & ns & 104.17 & ns & 104.17 & ns & 104.17 & ns \\
\hline $\begin{array}{l}\text { B2 vs B3 } \\
\text { Bioplus }\end{array}$ & 1 & $50.00 \mathrm{I}$ & ns & 112.50 & ns & 112.50 & ns & 112.50 & ns \\
\hline$B 1$ vs $B 23$ & 1 & $16.67 \mathrm{I}$ & ns & 104.17 & ns & 104.17 & ns & 104.17 & ns \\
\hline $\begin{array}{l}\text { B2 vs B3 } \\
\text { T0 vs }\end{array}$ & 1 & 0.00 & ns & 112.50 & ns & 112.50 & ns & 112.50 & ns \\
\hline Resto & 1 & $6.94 \mathrm{I}$ & ns & 4.44 & ns & 4.44 & ns & 4.44 & ns \\
\hline Error & 27 & 68.43 & & 42.59 & & 42.59 & & 42.59 & \\
\hline $\mathrm{CV} \%$ & & 30.27 & & 27.77 & & 27.77 & & 27.77 & \\
\hline Media & & 11.25 & & 23.50 & & 23.50 & & 23.50 & \\
\hline
\end{tabular}

ns: no significativo ( $p>0.05)$; ${ }^{*}$ significativo $(p<0.05) ;{ }^{* *}$ altamente significativo $(p<0.01)$ Productos: A1: Fosficarben; A2: Citrubact; A3: Bioplus; CV\%: Coeficiente de variación. Dosis: B1: Alta; B2: Media; B3: Baja.

La incidencia 2, 3 y 4 al utilizar Fosficarben fue de 20,00\%, la misma que es la más baja y difiere significativamente de los tratamientos Citrubact y Bioplus con los cuales se registraron 26,67 y 24,14\% (Tabla 4), esto quizá se deba a que el Fosficarben posee en su estructura un compuesto fosforado que influye con mayor eficacia el control de P. infestans. 
Sin embargo, productos como Bioplus y Citrubact también fueron eficaces en el control, Según: Agrios, 2002, el tizón tardío puede destruir totalmente todas las plantas de una zona de cultivo al cabo de una o dos semanas cuando las condiciones climáticas son favorables y cuando no se aplica ningún método de control. No obstante, las pérdidas varían de un área a la otra y de año en año dependiendo de la temperatura y la humedad predominantes en ciertos períodos de la estación de crecimiento de las plantas y de los métodos de control empleados.

\section{Table 4}

Comportamiento de la incidencia de P. infestans del cultivo de papa (S. tuberosum cV. Fripapa) bajo el efecto de tres productos ecológicos con tres dosis para el control de lancha según tukey al 5\%, en el cantón riobamba, provincia de Chimborazo.

\begin{tabular}{|c|c|c|c|c|c|c|c|c|c|c|c|}
\hline \multirow[b]{2}{*}{ Variables } & \multicolumn{3}{|c|}{ Productos } & \multirow[b]{2}{*}{ Sign } & \multicolumn{5}{|c|}{ Dosis } & \multirow[b]{2}{*}{$\mathrm{CV} \%$} & \multirow[b]{2}{*}{ Media } \\
\hline & Fosficarben & Citrubact & Bioplus & & Alta & Media & & Baja & Sign & & \\
\hline Incidencia (\%) I & $8.33 \mathrm{a}$ & $10.83 \mathrm{a}$ & $14.17 \mathrm{a}$ & ns & $9.17 \mathrm{a}$ & 13.33 & & $10.83 \mathrm{a}$ & ns & 73.53 & 11.25 \\
\hline Incidene: & $20.00 \mathrm{a}$ & $26.67 \mathrm{a}$ & $24.17 \mathrm{a}$ & ns & 20.83 a & 23.33 & & $26.67 \mathrm{a}$ & ns & 27.77 & 23.50 \\
\hline Incidenci & $20,00 \mathrm{a}$ & $26.67 \mathrm{a}$ & $24.17 \mathrm{a}$ & $\mathrm{n}$ & 20.83 a & 23.33 & & $26.67 \mathrm{a}$ & ns & 27.77 & 23.50 \\
\hline Incidencia (\%) 4 & $20.00 \mathrm{a}$ & $26.67 \mathrm{a}$ & $24.17 \mathrm{a}$ & ns & 20.83 a & 23.33 & & $26.67 \mathrm{a}$ & ns & 27.77 & 23.50 \\
\hline
\end{tabular}

Letras iguales no difieren significativamente según Tukey al 5\%.

ns: No significativo ( $p>0.05)$; ${ }^{*}$ significativo $(p<0.05)$; ${ }^{* *}$ altamente significativo $(p<0.01)$.

\subsection{Intensidad de P. Infestans}

La intensidad con la que se presentó $P$. infestans en el cultivo de papa en la primera lectura realizada a los 88 días después de la siembra fue de 2,60 con un coeficiente de variación de 30,96\% (Tabla 5), al someter los resultados experimentales al análisis de varianza se puede mencionar que no se registró diferencia estadística entre los diferentes tratamientos.

Este comportamiento puede deberse a que no se presentaron precipitaciones que den las condiciones requeridas de humedad para el desarrollo de la enfermedad. Según: Blitecast (2001), hay varias condiciones ambientales que propician la propagación de $P$. infestans.

Mediante el uso de los sistemas de pronóstico meteorológico, si se detecta que se van a producir las condiciones apropiadas para la extensión de la plaga, se recomienda el uso de fungicidas.

Un Periodo de Beaumont es un período de $48 \mathrm{hr}$ consecutivas, en el cual al menos en 46 de las lecturas de temperatura y humedad relativa de un determinado lugar no han sido menores de $20^{\circ} \mathrm{C}$ y $75 \%$, respectivamente (Blitecast, 2001).

Un Periodo de Smith es un período de al menos dos días consecutivos en el que la temperatura mínima es de $10^{\circ} \mathrm{C}$ o superior, y en cada día durante por lo menos $11 \mathrm{hr}$ la humedad relativa es superior al 90\% (Blitecast, 2001). 
Según la separación de medias con Tukey al 5\%, no presentaron diferencia significativa entre dosis a los 88 días después de la siembra.

La segunda lectura de intensidad de $P$. infestans a los 95 días de La siembra, en promedio fue de $4,15 \%$, y un coeficiente de variación de 18,72\% (Tabla 5), al someter los resultados experimentales al análisis de varianza presentó diferencia estadística al contrastar el tratamiento control y el resto de tratamientos alternativos puesto que presentaron una intensidad de 11,50 y $3,33 \%$ respectivamente, lo que permite mencionar que la utilización de productos para lancha es indispensable, si no se controla de alguna manera el cultivo de papa es muy susceptible a este tipo enfermedad.

\section{Table 5}

Cuadrados medios para la intensidad de P. infestans.

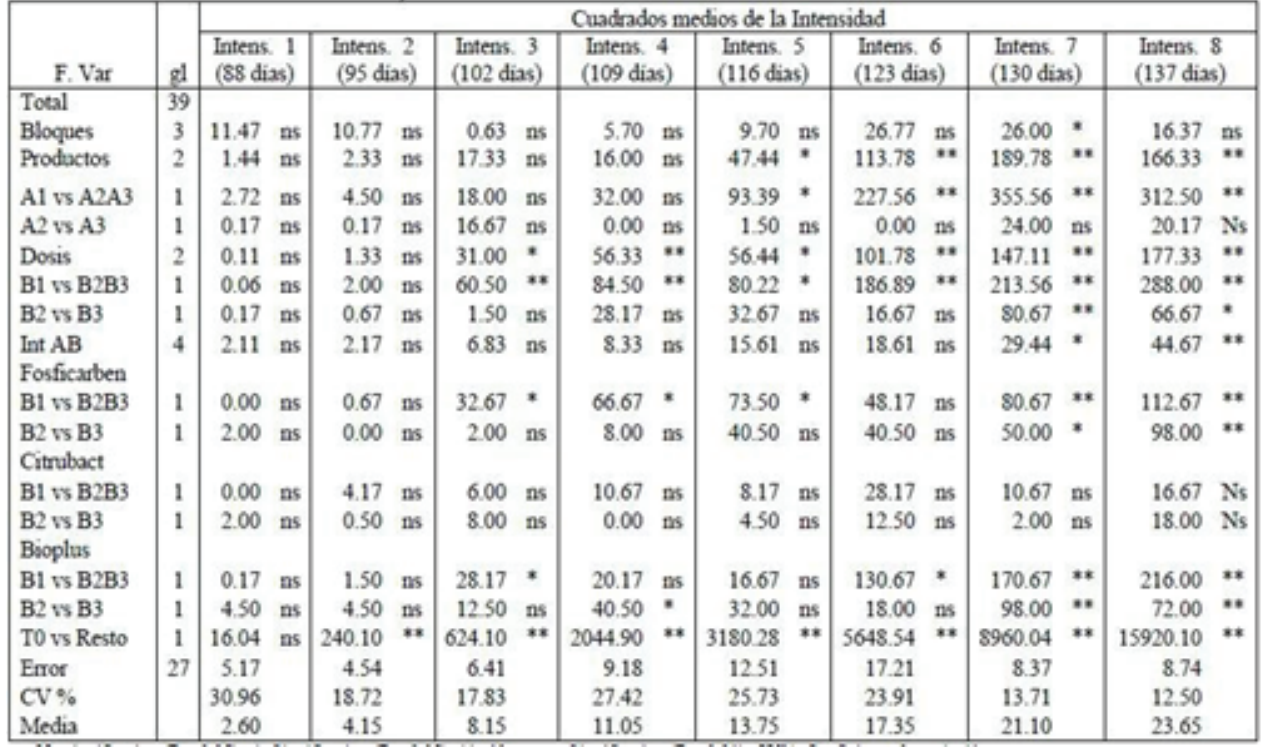

ns: no significativo ( $p>0.05) ;{ }^{*}$ significativo $(p<0.05)$; **altamente significativo $(p<0.01)$; CV\%: coeficiente de variación.

Productos: A1 vs A2A3: Fosficarben vs Citrubact y Bioplus; A2 vs A3: Citrubact vs Bioplus.

\subsection{Rendimiento}

La categoría pequeña (40-60 g y de 4 a $4,9 \mathrm{~cm}$ ), del cultivo de papa ( $\mathrm{S}$. tuberosum var. Fripapa), en promedio registró una media de $2,21 \mathrm{~kg}$ y un coeficiente de variación de 18,70\% (Tabla 6), al aplicar los resultados experimentales al análisis de varianza se determinó diferencias significativas entre dosis B1 vs B2B3, dentro del Citrubact B1 vs $\mathrm{B} 2 \mathrm{~B} 3$ y el control vs el resto de los tratamientos alternativos.

En todo el estudio prevalece la distinción entre dosis alta vs dosis media y baja, se registró un mayor número de tubérculos con estas características en las dosis media y 
baja de los productos; este calibre de tubérculo no tiene mucha posibilidad de ingresar al mercado (sólo como semilla) y su precio en general es bajo.

Según Tukey al 5\% la categoría pequeña, tuvo menos cantidad de tubérculos en el tratamiento A2B3, con 1,8 kg (Tabla 8). Este tratamiento está conformado por Citrubact en dosis baja, pero su máxima cantidad cavada en la parcela neta fue de 2,68 con Citrubact en dosis alta, sólo es esta parte del ensayo tenemos esta diferencia donde la dosis baja fue más eficaz.

\section{Table 6}

Cuadrados medios para la rendimiento del cultivo de papa.

\begin{tabular}{|c|c|c|c|c|c|c|c|c|c|c|c|}
\hline \multirow[b]{2}{*}{ F. Var } & \multirow[b]{2}{*}{ gl } & \multicolumn{10}{|c|}{ Cuadrados medios para los rendimientos } \\
\hline & & Pequeñ: & & Median & & Grande & & Gigan & & Total & \\
\hline Total & 39 & & & & & & & & & & \\
\hline Bloques & 3 & $0.52 *$ & * & 3.22 & ** & 2.65 & ** & 1.17 & * & 12.82 & ** \\
\hline Productos & 2 & $0.07 \mathrm{n}$ & ns & 7.90 & ** & 4.72 & $* *$ & 1.59 & ** & 21.90 & ** \\
\hline$A 1$ vs $A 2 A 3$ & 1 & $0.02 \mathrm{n}$ & ns & 0.67 & ns & 9.28 & ** & 2.87 & ** & 32.44 & ** \\
\hline$A 2$ vs $A 3$ & 1 & $0.13 \mathrm{n}$ & ns & 15.14 & ** & 0.15 & ns & 0.31 & ns & 11.36 & ** \\
\hline Dosis & 2 & $0.57 \mathrm{n}$ & ns & 30.61 & ** & 34.32 & ** & 1.95 & ** & 169.45 & ** \\
\hline $\mathrm{B} 1$ vs $\mathrm{B} 2 \mathrm{~B} 3$ & 1 & $1.13 *$ & * & 38.59 & ** & 63.94 & ** & 3.81 & ** & 296.58 & ** \\
\hline B2 vs B3 & 1 & $0.02 \mathrm{n}$ & ns & 22.64 & ** & 4.71 & *** & 0.09 & ns & 42.32 & ** \\
\hline $\begin{array}{l}\text { Int } A B \\
\text { Fosficarben }\end{array}$ & 4 & $0.25 \mathrm{n}$ & ns & 3.08 & $* *$ & 11.26 & ** & 1.58 & ** & 9.35 & ** \\
\hline $\mathrm{B} 1$ vs $\mathrm{B} 2 \mathrm{~B} 3$ & 1 & $0.24 \mathrm{n}$ & ns & 21.43 & ** & 28.95 & $* *$ & 3.27 & ** & 151.60 & $* *$ \\
\hline $\begin{array}{l}\text { B2 vs B3 } \\
\text { Citrubact }\end{array}$ & 1 & $0.20 \mathrm{n}$ & ns & 5.81 & ** & 15.85 & $* *$ & 0.06 & ns & 32.32 & ** \\
\hline $\mathrm{B} 1$ vs $\mathrm{B} 2 \mathrm{~B} 3$ & 1 & $1.59 *$ & $* *$ & 0.62 & ns & 2.28 & $*$ & 4.56 & ** & 32.39 & ** \\
\hline $\begin{array}{l}\text { B2 vs B3 } \\
\text { Bioplus }\end{array}$ & 1 & $0.11 \mathrm{n}$ & ns & 10.26 & ** & 9.75 & $* *$ & 0.76 & ns & 1.64 & ns \\
\hline$B 1$ vs $B 2 B 3$ & 1 & $0.01 \mathrm{n}$ & ns & 28.54 & ** & 48.45 & ** & 0.32 & ns & 139.83 & ** \\
\hline B2 vs B3 & 1 & $0.01 \mathrm{n}$ & $\mathrm{ns}$ & 6.90 & $* *$ & 8.40 & ** & 1.26 & $*$ & 18.51 & ** \\
\hline T0 vs Resto & 1 & $3.21 *$ & ** & 155.92 & ** & 122.63 & ** & 41.40 & ** & 795.37 & ** \\
\hline Error & 27 & 0.17 & & 0.48 & & 0.51 & & 0.27 & & 0.84 & \\
\hline CV \% & & 18.70 & & 8.81 & & 9.47 & & 10.70 & & 4.09 & \\
\hline Media & & 2.21 & & 7.83 & & 7.57 & & 4.81 & & 22.41 & \\
\hline
\end{tabular}

ns: no significativo ( $p>0.05)$; * significativo ( $p<0.05)$; ${ }^{* *}$ altamente significativo $(p<0.01)$; CV\%: coeficiente de variación.

Productos: A1 vs A2A3: Fosficarben vs Citrubact y Bioplus; $A 2$ vs A3: Citrubact vs Bioplus. Dosis: B1 vs B2B3: Alta vs Media y Baja; B2 vs B3: Media vs Baja.

La categoría mediana (61-80 g y de $5-5,9 \mathrm{~cm}$ ), del cultivo de papa (S. tuberosum var. Fripapa), en promedio registró una media de 7,83 kg y un coeficiente de variación de 8,81 \% (Tabla 6), al aplicar los resultados experimentales al análisis de varianza se determinó diferencia significativa entre productos $A 2$ vs $A 3$, entre dosis $B 1$ vs $B 2 B 3$ y B2 vs B3, existe interacción ab, dentro de Fosficarben B1 vs B2B3 y B2 vs B3, dentro del Citrubact B2 vs B3, dentro de Bioplus B1 vs B2B3 y B2 vs B3 y el control vs el resto de tratamientos alternativos.

La categoría grande (81-100 g y de 6-6,9 cm), del cultivo de papa (S. tuberosum var. Fripapa), en promedio registró una media de 7,57 kg y un coeficiente de variación 
de 9,47\% (Tabla 6), al aplicar los resultados experimentales al análisis de varianza se determinó diferencia significativa entre productos $A 1$ vs $A 2 A 3$, entre dosis $B 1$ vs $B 2 B 3$ y $B 2$ vs B3, existe interacción ab, dentro de Fosficarben B1 vs B2B3 y B2 vs B3, dentro del Citrubact B1 vs B2B3 y B2 vs B3, dentro de Bioplus B1 vs B2B3 y B2 vs B3 y el control vs el resto de tratamientos alternativos.

El promedio en la categoría grande con $7,57 \mathrm{~kg}$ no tiene mucha diferencia con la categoría mediana de 7,83\%, esto es importante porque la categoría grande es muy apreciada en el mercado y llega a tener buen precio (dependiendo siempre de la demanda).

La categoría gruesa (101-120 g y de 7-8 cm), del cultivo de papa (S. tuberosum var. Fripapa), en promedio registró una media de $4,81 \mathrm{~kg}$ y un coeficiente de variación de 10,70\% (Tabla 6), al aplicar los resultados experimentales al análisis de varianza se determinó diferencia significativa entre productos $A 1$ vs $A 2 A 3, A 2$ vs $A 3$ entre dosis $B 1$ vs B2B3 y B2 vs B3, existe interacción ab, dentro de Fosficarben B1 vs B2B3 y B2 vs $B 3$, dentro del Citrubact B1 vs B2B3, dentro de Bioplus B1 vs B2B3 y B2 vs B3 y el control vs el resto de tratamientos alternativos.

El promedio en la categoría gruesa con $4,81 \mathrm{~kg}$ (Tabla 6) es menor con respecto a la categoría mediana y grande, ésta categoría es la más apreciada en el mercado para negocios que preparan papas a la francesa debido a su textura, su precio no depende en gran medida de la demanda porque ya se puede tener clientes fijos.

\section{Table 7}

Comportamiento del rendimiento del cultivo de papa.

\begin{tabular}{|c|c|c|c|c|c|c|c|c|c|c|}
\hline \multirow[b]{2}{*}{ Variables } & \multicolumn{3}{|c|}{ Fosficarben } & \multicolumn{3}{|c|}{ Citrubact } & \multicolumn{3}{|c|}{ Bioplust } & \multirow[b]{2}{*}{$\begin{array}{c}\text { Sig } \\
\mathrm{a}\end{array}$} \\
\hline & Ale & $\begin{array}{l}\text { Medi } \\
\text { a }\end{array}$ & Baja & Ale & \begin{tabular}{|l} 
Medi \\
\end{tabular} & Baja & Alta & $\begin{array}{l}\text { Medi } \\
\text { a }\end{array}$ & Baja & \\
\hline $\begin{array}{c}\text { Rendimiento categoria } \\
\text { pequera }\end{array}$ & $2,35 \mathrm{a}$ & 1,88 a & $2,20 \mathrm{a}$ & $2.68=$ & $2,03 \mathrm{a}$ & $1,80 \mathrm{a}$ & $2,06 \mathrm{a}$ & $1,97 \mathrm{a}$ & $2.04 a$ & as \\
\hline $\begin{array}{c}\text { Rendimiento categoria } \\
\text { mediana }\end{array}$ & $\begin{aligned} & 10,5 \mathrm{a} \\
& 7 \mathrm{~b}\end{aligned}$ & & $6.88 \mathrm{~d}$ & 792 d & & $6.30 \mathrm{~d}$ & 11.3 & $9.02 \stackrel{b}{\mathrm{c}}$ & $7.16 \mathrm{~d}$ & ** \\
\hline Rendimiento categoria & 11,0 & & & & & & 10,7 & & & \\
\hline $\begin{array}{c}\text { grande } \\
\text { Rendimiento categoria }\end{array}$ & 7 a & $\begin{aligned} & 9,18 \mathrm{c} \\
& \mathrm{b}\end{aligned}$ & $\begin{array}{rl}6,36 & d \\
b\end{array}$ & $8,33 \mathrm{c}$ & $\begin{array}{rl}6,30 & d \\
b\end{array}$ & $8,51 \mathrm{c}$ & $1 \mathrm{~b}$ & $7,48 \mathrm{~d}$ & $\begin{aligned} & 5,43 \mathrm{~d} \\
& \mathrm{a}\end{aligned}$ & $*$ \\
\hline gruesa & $\begin{array}{l}6.29 \mathrm{a} \\
30.2\end{array}$ & $5.09 \mathrm{c}$ & $\begin{array}{l}5.27 \mathrm{c} \\
20.7\end{array}$ & $\begin{array}{l}5.94 \mathrm{~b} \\
24.8\end{array}$ & $4.94 \mathrm{c}$ & $\begin{array}{l}4,32 \mathrm{c} \\
20.9\end{array}$ & $\begin{array}{l}4,61 \mathrm{c} \\
28.7\end{array}$ & $4,56 \mathrm{c}$ & $\begin{array}{l}5.35 \text { b } \\
19.9\end{array}$ & $*$ \\
\hline Rendimiento total & $7 a$ & $24,74 \mathrm{c}$ & $2 d$ & $6 \mathrm{c}$ & $21.83 \mathrm{~d}$ & $3 \mathrm{~d}$ & $4 b$ & $23,02 \mathrm{C}$ & $8 e$ & $*$ \\
\hline
\end{tabular}

Letras iguales no difieren significativamente según Tukey al $5 \%$.

ns: no significativo ( $p>0.05)$; ${ }^{*}$ significativo $(p<0.05) ;{ }^{* *}$ altamente significativo $(p<0.01)$

La diferencia estadística en la dosis no fue significativa para la dosis alta, para la dosis media hubo una diferencia en la categoría mediana, grande, gruesa y en el rendimiento total, ocurriendo lo mismo en la dosis baja (Tabla 7).

Según Tukey al 5\%, presentó diferencia significativa en Fosficarben en dosis alta en la categoría mediana, diferencias en dosis media en la categoría mediana, grande, gruesa y en el rendimiento total, presentando el mismo comportamiento en la dosis baja (Tabla 7). 
La diferencia en Citrubact fue significativa en la dosis alta, media y baja en la categoría mediana, grande, gruesa y en el rendimiento total. Bioplus presentó diferencia estadísticas en la dosis alta en la categoría grande, gruesa y en el rendimiento total, mientras que en la dosis media y baja presentó diferencias en las cuatro categorías y en el rendimiento total (Tabla 7).

\subsection{Análisis Económico}

Según: Pérez (2001) el manejo de tizón tardío es uno de los pasos más costosos entre las actividades que se deben realizar antes, durante el cultivo y en la etapa de poscosecha. El manejo integrado de $P$. infestans incluye una serie de medidas, entre las cuales tenemos el control genético, químico y cultural.

En la Tabla 8, se puede apreciar que el mayor rendimiento en $\mathrm{kg} / \mathrm{ha}$ lo obtiene el Tratamiento 1 de Fosficarben en dosis alta (4 cc/ltro), con $21496 \mathrm{~kg}$, con un costo variable de 639,20 USD, seguido de cerca por el tratamiento 7 de Bioplus en dosis alta (5 cc/ltro) con un rendimiento de $20413,71 \mathrm{~kg}$ y un costo variable de 443,85 USD, por tanto el beneficio neto de Fosficarben en dosis alta (4 cc/ltro) es de 3423,70 y de Bioplus en dosis alta (5 cc/ltro) es de 3414,34.

En el análisis de dominancia (Tabla 9), los tratamientos no dominados son T1: Fosficarben en dosis alta (4 cc/ltro), T7: Bioplus en dosis alta ( $5 \mathrm{cc} /$ Itro), T8: Bioplus en dosis media (4 cc/ltro), T6: Bioplus en dosis baja ( $3 \mathrm{cc} /$ Itro), T0: testigo, esto quiere decir que presentaron menos costo y mayor beneficio, pero de todos éstos tratamientos podemos destacar que el tratamiento 1 de Fosficarben en dosis alta (4cc/ltro), es el que produce un mayor beneficio neto con 3423,70 USD, sin embargo la tasa de retorno marginal es apenas del $4,76 \%$, siendo la más baja del resto de tratamientos, esto se debe a que su costo es el más elevado.

El tratamiento que obtuvo la mayor tasa de retorno fue el tratamiento 6 de Citrubact en dosis baja (1 cc/ltro) con 798,97\%, seguido por Bioplus en dosis media (4 cc/ltro) con una tasa de retorno marginal de $765,14 \%$.

\section{Conclusiones}

El producto ecológico más eficaz para el control de lancha ( $P$. infestans) en el cultivo de papa (S. tuberosum cv. Fripapa) fue Fosficarben, presentando un porcentaje de incidencia del $20 \%$, frente a $26,67 \%$ de Citrubact y $24,17 \%$ de Bioplus, con un coeficiente de varianza de $27,77 \%$ y una media de $23,50 \%$ que no fueron significativas, sin embargo en intensidad hubo diferencias significativas entre productos teniendo Fosficarben un porcentaje de intensidad de 12,83\%, para Citrubact de 18,17 y para Bioplus de 20,00\%, 
Table 8

Costos que varían por hectárea de los tratamientos.

\begin{tabular}{l|ll|l|l|l} 
Productos & Dosis & & $\begin{array}{l}\text { Costo por } \\
\text { aplicación }\end{array}$ & $\begin{array}{l}\text { Número de } \\
\text { aplicacion es }\end{array}$ & $\begin{array}{l}\text { Costo } \\
\text { Variable }\end{array}$ \\
\hline Fosficarben & $4 \mathrm{cc} /$ litro & ALTA & 127,84 & 5 & 639,2 \\
\hline $3 \mathrm{cc} /$ litro & & MEDIA & 95,88 & 5 & 479,4 \\
\hline 2cc/litro & & BAJA & 63,92 & 5 & 319,6 \\
\hline Citrubact & $3 \mathrm{cc} /$ litro & ALTA & 106,53 & 5 & 532,65 \\
\hline 2 cc/litro & & MEDIA & 71,02 & 5 & 355,1 \\
\hline 1 cc/litro & & BAJA & 35,51 & 5 & 177,55 \\
\hline Bioplus & $5 \mathrm{cc} /$ litro & ALTA & 88,77 & 5 & 443,85 \\
\hline $4 \mathrm{cc} /$ litro & & MEDIA & 71,02 & 5 & 355,1 \\
\hline $3 \mathrm{cc} /$ litro & & BAJA & 53,27 & 5 & 266,35 \\
\hline
\end{tabular}

Fuente: Autor.

Table 9

Análisis de dominancia.

\begin{tabular}{|c|c|c|c|}
\hline Tratamientos & $\begin{array}{c}\text { Beneficio } \\
\text { neto } \\
\text { usd/ha }\end{array}$ & $\begin{array}{l}\text { Costo } \\
\text { Variable }\end{array}$ & $\begin{array}{c}\text { Análisis de } \\
\text { Dominancia }\end{array}$ \\
\hline T1 & 3423,70 & 639,20 & ND \\
T7 & 3414,34 & 443,85 & ND \\
T2 & 2841,19 & 479,40 & D \\
T4 & 2804,72 & 532,65 & D \\
T8 & 2735,28 & 335,10 & ND \\
T6 & 2631,28 & 177,55 & ND \\
T5 & 2575,21 & 355,10 & D \\
T3 & 2461,37 & 319,60 & D \\
T9 & 2415,62 & 266,35 & D \\
T0 & 1212,71 & 0 & ND \\
\hline
\end{tabular}

Fuente: Autor.

siendo una diferencia altamente significativa con un coeficiente de varianza de 12,50\% y una media de $23,65 \%$.

La dosis más eficaz para el control de lancha ( $P$. infestans) en el cultivo de papa (S. tuberosum cv. Fripapa) fue la dosis alta con una incidencia de $20,83 \%$, seguida por la dosis media con un porcentaje de incidencia de $23,33 \%$ y la dosis baja con $26,67 \%$, con un coeficiente de varianza de $27,77 \%$ y una media de 23,50 . En intensidad si presentó diferencia altamente significativa entre dosis con un porcentaje de intensidad para la dosis alta de 10,17\%, 14,17\% para la dosis media y 15,83\% para la dosis baja, con un coeficiente de varianza de $25,73 \%$ y una media de 13,75 , el producto más eficaz fue 


\section{Table 10}

Tasa de retorno marginal de los tratamientos.

\begin{tabular}{|c|c|c|c|c|c|}
\hline Tratamientos & $\begin{array}{c}\text { Beneficio } \\
\text { neto } \\
\text { usd/ha }\end{array}$ & $\begin{array}{c}\text { Beneficio } \\
\text { Neto } \\
\text { Marginal }\end{array}$ & $\begin{array}{c}\text { Costo } \\
\text { Variable } \\
\text { Productos }\end{array}$ & $\begin{array}{c}\text { Costo } \\
\text { Variable } \\
\text { Marginal }\end{array}$ & $\begin{array}{c}\text { Tasa } \\
\text { Marginal } \\
\text { de retorno } \\
\%\end{array}$ \\
\hline T1 & 3423,70 & 9,36 & 639,20 & 195,35 & 4,76 \\
T7 & 3414,34 & 679,06 & 443,85 & 88,75 & 765,14 \\
T8 & 2735,28 & 104,00 & 355,10 & 177,55 & 58,58 \\
T6 & 2631,28 & 1418,57 & 177,55 & 177,55 & 798,97 \\
T0 & 1212,71 & & 0 & & \\
\hline
\end{tabular}

Fuente: Autor.

Fosficarben con $4 \mathrm{cc} / \mathrm{lt}$, que presentó la menor incidencia e intensidad del patógeno además del más alto rendimiento, destacándose del resto de dosis y productos.

Dentro del análisis económico, hay que destacar que la dosis baja de Citrubact fue la que mejor tasa marginal de retorno con $798,97 \%$, con un beneficio neto de 2631,28 USD y un costo variable de 177,55 USD, debido a su dosis baja, le permite ser el más rentable de los tratamientos en estudio.

\section{References}

[1] FAO, 2011. Tesoro enterrado: la papa.

[2] INEC. 2011. Instituto Nacional de Estadísticas y Censos.

[3] AGRIOS, G. 2002. Fitopatología. Phytophtora infestans. Editorial Limusa, S.A. Grupo Noriega Editores. Segunda edición. México. pág. 837

[4] SCHAWINN, F. 1995. New Developments in Chemical Control of Phytophtora infestans. In "Phytophtora is Biology, taxonomy, Ecology and Pathology" (D. Erwin, S. Bartnicki y P. Tsao, eds.),pp327-334. The American Phytopathologycal Society, Minnesota. US.

[5] SUQUILANDA, M. 2011. Producción Orgánica de Cultivos Andinos. Cultivo orgánico de papa. Quito, Ecuador. Ministerio de Agricultura, UNOCANC, FAO. 348p

[6] NEIRA, R. 1986. Tecnología del cultivo de papa. En memorias del IV Curso sobre tecnología del cultivo y manejo de la semilla de papa. Quito, Ecuador, 16 al 27 de Octubre de 1978. Pp 21-32

[7] ALVIAR, C. 2004 Manual de agricultura alternativa: principios. pp. 96 
[8] AGROBEST. S.A. 2012. Agricultura orgánica, biológica, ecológica, sustentable.

[9] BLITECAST BY DR MACKENZIE, 1981. Scheduling fungicide applications for potato late blight with Plant Disease Volume 65 pages 394-399

[10] CALDERONI, A. 1978. "Enfermedades de la papa y su control. Editorial Hemisferio Sur. Buenos Aires, Argentina. 43p.

[11] CESTTA, 2012. Análisis Centro de Servicios Técnicos y Transferencia de Tecnología Ambiental

[12] CIPOTATO.ORG (En línea), 2012 http://cipotato.org/region-quito/informacion/ inventario-de-tecnologias/tesis

[13] EWIN, D. Y RIBEIRO, O; 1996. Chapter 33: Phytophtora infestans (Mont.) de Bary (1876). In "In Phytophtora infestans Diseases Worldwide". 353p. The American Phytopathological Society. Minesota. US.

[14] GONZALES, L.; 1997. Hongos Fitopatógenos. In “Introducción a la Fitopatología” (M. de la Cruz, ed), pp 16-23. Instituto Intermamericano de Ciencias Agrícolas, San José. CR.

[15] HIDALGO, L. 2011. Generalidades del Bioplus. 74

[16] HUAMÁN, Z. 1986 "Botánica Sistemática y morfología de la papa” 2 edición rev/Ed. Centro Internacional de la papa, Lima. 22 p

[17] INIAP, 2013. http//www.iniap.gob.ec//Información\{\%\}20Técnica\{\%]20de\{\%\}20la\{\%]20varieda

[18] KOEPSELL, P. 1994 Pacific Northwest Plant Disease Control Handbook, Oregon State University Press: $\mathrm{p} 165$.

[19] KROMMAN, P. 2007. Improving potatoe late blight control strategies for resoursepoor farmers in low in-put agriculture in Andean Ecuador. Tesis, University of Copenhagen

[20] MONTESDEOCA, F. 2005. Guía para la producción, comercialización y uso de semilla de papa de calidad. Quito. PNRT-INIAP- Proyecto Fortipapa. 40 p.

[21] MUÑOZ, M. 2010. Instructivo: Prospección de cultivos y Proyectos. Sag - Chile.

[22] MUÑOZ, F. Y CRUZ, L. 1984. Manual del cultivo de papa. Quito. INIAP. 44p.

[23] OREGONSTATE.EDU (En línea), 2012 http//extensión.oregonstate.edu

[24] ORGANICO \& NATURAL (En línea), 2012 http://organicoynatural.cl75

[25] OYARZUN, P. 2002. Manejo Agronómico. El cultivo de papa en Ecuador. Quito. INIAP, CIP. Pp $51-82$.

[26] PEREZ, A. Phytophtora infestans (Mont.) de Bary: control mediante fungicidas e inductores de resistencia química en papa. Tesis. Universidad Nacional Agraria La Molina, Lima, PE.

[27] PHARMACY.EDU (En línea), 2012 http://suprfund.pharmacy.edu 
[28] ínea base de variedades de papa en comunidades de las provincias de Carchi, Chimborazo y parroquia Quero en Ecuador. 83p. Instituto Nacional de Investigaciones Agropecuarias.

[29] POLLOCK, M. 2003. HORTALIZAS. Cultivo de Papa; Editorial BLUME. Primera edición en lengua española 2003.Barcelona-España. pág. 272

[30] PUMISACHO, M.; SHERWOOD, S. 2002. El cultivo de la papa en Ecuador. Primera edición (INIAP) Quito-Ecuador. Pág 2

[31] PUMISACHO, M. Y VELASQUEZ, J. 2009. Manual del cultivo de papa para pequeños productores. Quito. INIAP, COSUDE. 98p.

[32] REDALYC.MX (En línea), 2012 http://redalyc.uaemex.mx

[33] ROBERTSON, N. 1991. The Challenge of Phytophthora infestans. Advance of Plant Pathology, Vol 7: Phytophthora infestans: The cause of Ligt Blight of Potato. (D.S. Ingram. P.H. Williams, eds). Academia Press, San Diego, USA.

[34] TAPIA, M. 1993. Vision general y características del Agroecosistema Andino. Memorias del Taller Internacional sobre Ecosistema Andino, pp 51 - 56. International potato Center. Lima.

[35] TURKESTEEN. LJ and FLIER, WG. 2002b. Late blight: Its Global Estatus in 2002 and Beyond. Proceedings of the Global iniciative on Late Blight Conference, 11-13 July, Hamburg, Germany. P 1-9.

[36] UQUILLAS, J.; CRISSMAN, CH. PETERSON, W. 2002 "La papa en los sistemas de Producción agropecuaria de la sierra ecuatoriana". FUNDAGRO. Quito, Ecuador. 38p.

[37] ZENTMYER, G. 1985. The world of Phytophthora. Páginas 1-7. En: Phytophthora: Its Biology, Taxonomy, Ecology, and Pathology. Eds. D. C. Ewin,S. Bartnick Garcia, P. H. Tsao. U.S.A. 